\title{
Demand Planning \& Control - Handling Multiple Perspectives Through a Holistic Approach to Hierarchical Planning
}

\author{
Peter Nielsen and Kenn Steger-Jensen \\ Aalborg University, Department of Production, Fibigerstraede 16, 9220 \\ Aalborg, Denmark, \\ Centre of Supply Chain Integration, WWW home page: \\ http://www.misg.aau.dk
}

\begin{abstract}
Hierarchical Demand Planning (HDP) is an intricate part of most companies today. HDP is based on the assumption of independence among variables, and this allows for simple and easy aggregation and separation of plans and data. However, the most commonly used arguments for grouping and subsequent aggregating is shared traits contrary to the assumption of independency. One of the predominant issues is the conflicting objectives on different decision levels. An example of this is found in hierarchical forecasting of demand. When forecasting on e.g. a product family level to establish capacity requirements, the objective is usually to achieve a Mean Error (ME) of zero. This conflicts with forecasting for Demand Planning (DP) purposes on SKU level, where minimization of the Standard Deviation of Error (SDE) might be more important. In this paper these issues are addressed through a simple example of hierarchical forecasting and use of a Goal Programming (GP) approach to satisfy both objectives. It is found that some general guidelines for handling multiple objectives within HDP can be inferred from this, leading the way for a holistic demand planning framework.
\end{abstract}

\section{Introduction}

Companies today find themselves facing: Short Product Life Cycles, complex products, supply networks as well as rising demands for profitability. As a result the Demand Planning and Control (DPC) situation is highly complex and dynamic. This sets new standards for companies' ability to; share information internally and in their supply network, quickly arrive at an "optimal" plan, execute the plan and take corrective measures as necessity dictates. Due to the varying nature of manufacturing companies with regards to products and competitive priorities, the DPC processes and objectives vary greatly between companies and planning areas. Typically manufacturing companies have a need to plan on several levels, i.e. a planning

Please use the following format when citing this chapter:

Nielsen, P. and Steger-Jensen, K., 2008, in IFIP International Federation for Information Processing, Volume 257, Lean Business Systems and Beyond, Tomasz Koch, ed.; (Boston: Springer), pp. 57-65. 
hierarchy is present. Several different approaches to handle this have been presented, and the most important among them is the Hierarchical Demand Planning Approach (HDPA) $[1,2,8]$. However the HDPA has some weakness, among these the problem of feasibility and optimality of plans. Furthermore, several planning objectives exist and these need not be coherent. One approach to handling these issues of conflicting objectives would be a holistic demand planning framework where goals for satisfying a number of the objectives were achieved.

\section{Literature review}

A literature review of HDPA, hierarchical forecasting and Goal Programming (GP) was conducted.

A descriptive HDPA was first presented by Hax and Meal [8] to give an easy planning algorithm that would yield plans for several planning levels through a number of steps, starting with an aggregate plan. In many situations, the hierarchical planning (HPA) paradigm has been and continues to be a suitable and satisfactory framework for structuring the management tasks (e.g. planning and controlling). Bitran et al. [1], Bitran and Hax [3], Hax and Golovin [7] and Hax and Meal [8] are the major pioneers in the development of HPA models. The success of the HDPA has been so widespread that certain authors recommend the approach for almost any medium-to-large scale situation $[13,15]$. Today, HDP is widely implemented in companies and supported by major ERP-system providers such as Oracle and SAP. However the issue of feasibility of the plans has not been completely addressed. The issues of feasibility and optimally of plans on different planning levels are negated by the assumption of independence between e.g. products with different product families. However, in reality dependencies exist, often due to the modularity of products, which gives a need for a new approach. The main reason for using the HDPA is that it delivers a plan with a minimum of processing; feasibility and optimality are however not guaranteed. A main issue is how (within a simple framework) to handle multiple planning objectives on multiple planning levels. Hax and Meal [8] use an absolute priorities approach and suggests optimizing for one objective (cost minimization) on an aggregate level and then to use this as a constraint on lower planning levels. There is of course ongoing discussions about which decisions are found at each level, which will be dealt with later, and this can depend on the type of manufacturing being conducted (e.g. Hendry and Kingsman [9]) and the degree of decentralization, but the general approach to sequential decision division is followed. In the original Hax-Meal system it is possible to obtain a feasible solution to the aggregate problem that will not permit any feasible solution to the subsequent family and individual-item scheduling problem, because of the aggregation at the production planning stage. Bitran et al. [2] suggest aggregating products with similar production costs, inventory costs and seasonal demand to product types. But an applicable definition of "similar" is lacking. Furthermore, the grouping of products based on similar attributes wills inevitable conflict with the main assumption of the HDPA, i.e. independency of demand. 
Hierarchical forecasting can be conducted in a number of ways, some methods concern forecasting on an aggregate level and on a lower level and then use a combination of methods arrive at a better lower level forecast [5, 14]. This however defeats on the major advantages associated with using aggregate data namely it is simpler, quicker and exhibits good qualities regarding variance. When focusing on fitting forecasts to a demand pattern, the regression techniques used ensure that $\mathrm{ME}=0$ and the noise is n.i.d. In practice many companies use simple times series techniques either due to the cost of using more complex techniques or due to limited capabilities within the company. These techniques do not always achieve a ME equal to zero. Firstly, many companies only forecast on aggregate data (e.g. product family demand data) and use simple distribution keys to arrive at lower level forecasts, which often is the approach supported by ERP and APS vendors. Secondly, a ME of zero on aggregate forecasts does not explicitly correspond to a low SDE on the disaggregate level when independency between product demands is present. This conflicts with the needs for data performance in the HDPA. I.e. the issue is how to balance an objective of $\mathrm{ME}=0$ on an aggregate forecast and still get as low a SDE on a lower level as possible since both are used as input to the HDP process.

Goal Programming (GP) $[4,10,11]$ is an approach to multiple objective scenarios in which each of the objectives has a target or goal. Its distinguished feature is that the objectives can be started as minimizing deviations from prespecified goals. GP differs from other optimization methods through the distinguishing between hard and soft constraints. Soft constraints are particularly well suited to address the real life situations encountered as in DP. The search strategies used are related to those used when solving mixed integer programming (MIP) problems via branch-and-bound procedures. In most MIP problems, constraints represent limitations or requirements, which must be met. The solution to a MIP problem does not allow a constraint to be violated. Thus, a way of modeling soft constraints is needed. Basically, there are two approaches to integer GP, which are called non-preemptive and preemptive. Non-preemptive is based on a weighted GP approach, where the goals are of roughly comparable importance, and the preemptive is based on a hierarchy of priority levels of the goals. Here, the weighted GP using mixed-integer variables (G-MILP) as in MIP will be used, since it is suitable for representing decision problems of the type encountered here.

\section{Goal programming applied to hierarchical forecasting using simple times series}

In this section, forecasting an example of the problems inherent in both neglecting the existence of diverging objectives and the dependency when utilizing the HDPA.

A product family of three products was used to exemplify the issue. For all three products 25 periods of demand existed. The three products have respectively: a constant demand (Product I), a linearly decreasing (Product II) and a linearly increasing trend (Product III). All had a stationary demand of 150 units/periods and a random element that is n.i.i.d. with mean zero and variance $\sigma^{2}$ of $10^{2}$. Demand for 
product II decreases with the same rate as demand for product III increases - a typical occurrence when a new product is taking over from an old product. This is an important issue when grouping items for aggregation purposes.

To use a simple approach the demand data was aggregated and four simple time series forecasting methods were used on the data. These were: the naive, simple moving average using six periods of data, exponential smoothing using an $\alpha$ of 0.3 and exponential smoothing with trend were the smoothing constants using HoltWinters' approach [13]. A six month moving average of demand for the individual products relative to the product family demand was used to find the distribution keys giving individual product forecasts. In the researchers experience with both medium and large sized companies this is a typical approach to get a SKU level forecast. The results with regards to $\mathrm{ME}, \mathrm{SDE}$ and MAPE are shown in table 1.

Table 1. The table shows respectively, the forecast on aggregated data, three disaggregated forecasts for Products I-III and an average of the performance of the disaggregated forecasts performance. Values marked with bold are used in the subsequent optimization.

\begin{tabular}{|l|c|c|c|c|c|c|c|c|}
\cline { 2 - 10 } & \multicolumn{2}{c|}{} & \multicolumn{2}{c|}{$\begin{array}{c}\text { Moving Average } \\
\text { Naive }(\mathrm{j}=1)\end{array}$} & \multicolumn{2}{c|}{$\begin{array}{c}\text { Exponential } \\
\text { smoothing }(\mathrm{j}=3)\end{array}$} & \multicolumn{2}{c|}{$\begin{array}{c}\text { Exponential } \\
\text { smoothing with } \\
\text { trend }(\mathrm{j}=4)\end{array}$} \\
\cline { 2 - 10 } & $\mathrm{ME}$ & $\mathrm{SDE}$ & $\mathrm{ME}$ & $\mathrm{SDE}$ & $\mathrm{ME}$ & $\mathrm{SDE}$ & ME & SDE \\
\hline Aggregate Forecast & $\mathbf{0 , 3}$ & 21,0 & $\mathbf{- 0 , 7}$ & 19,4 & $\mathbf{0 , 7}$ & 17,7 & $\mathbf{0 , 9}$ & 21,2 \\
\hline Product I & $-0,1$ & $\mathbf{1 1 , 0}$ & $-1,1$ & $\mathbf{9 , 6}$ & $-0,6$ & $\mathbf{9 , 4}$ & $-0,6$ & $\mathbf{9 , 9}$ \\
\hline Product II & 8,0 & $\mathbf{1 3 , 2}$ & 7,5 & $\mathbf{1 4 , 1}$ & 7,7 & $\mathbf{1 3 , 6}$ & 7,9 & $\mathbf{1 4 , 3}$ \\
\hline Product III & $-6,7$ & $\mathbf{1 0 , 1}$ & $-7,1$ & $\mathbf{9 , 0}$ & $-7,4$ & $\mathbf{8 , 8}$ & $-6,4$ & $\mathbf{9 , 7}$ \\
\hline
\end{tabular}

The approach to use distribution keys when dependency exists between sales of some products giving, as one would expect, a constant undershoot and overshoot of forecast on the disaggregated level. This of course runs contrary to good DP procedures. However, in a practical environment a constant over-/undershoot of forecast might not be nearly as costly as a high SDE, since the latter is used to establish service level - yet again it is recognized that the service level typically assumes noise to be n.i.d. with a mean of zero. Firstly, this is only relevant when the variance of the noise is small compared to the numerical deviation from $\mathrm{ME}$ equaling zero. Secondly, in a practical application shifts in demand patterns will typically result in some deviations from this assumption, due to e.g. sales discount.

It is recognized that some companies uses more complex forecasting techniques than those presented above. These would almost all use some form of regression approach to achieve $\mathrm{ME}=0$. However, this would only enhance the problem described, since no explicated relationship exists between ME and SDE when trend is present on the product level. And since multiple conflicting objectives exist on different planning levels some method to create a balance between these objectives is needed.

The next step was to use GP to balance performance on both planning levels, i.e. minimize $\mathrm{SDE}$ on $\mathrm{SKU}$ level and solving $\mathrm{ME} \approx 0$ on the aggregate level. 
The following variables are needed:

$\mathrm{x}_{\mathrm{j}}=$ Binary decision variable to decide whether to use a given aggregate forecast $j$ indicates the forecast type (e.g. naïve $j=1$ )

$\mathrm{w}_{\mathrm{k}} \quad=$ Weight of goal $k$

$\mathrm{d}_{\mathrm{j}^{+}}=$Deviation variable, $\mathrm{ME}>0$, for aggregate forecast $j$ on aggregate level

$\mathrm{d}_{\mathrm{j}^{-}} \quad=$ Deviation variable, $\mathrm{ME}<0$, for aggregate forecast $j$ on aggregate level

$\mathrm{d}_{\mathrm{ji}}{ }^{+}=$Deviation variable indicating deviation from lowest $\operatorname{SDE}\left(\operatorname{SDE}_{\min , \mathrm{i}}\right)$ for product $i$ using forecast $j$ on aggregate data

$\mathrm{ME}_{\mathrm{j}}=\mathrm{ME}$ of aggregate forecast using forecast type $j$

$\mathrm{SDE}_{\mathrm{ji}}=\mathrm{SDE}$ of product $i$ using forecast type $j$ on aggregate level

The decision variable $\mathrm{d}_{\mathrm{ji}}$ - needs not be defined since $\mathrm{SDE}_{\mathrm{min}, \mathrm{i}}$ is by definition the minimum. The decision variables $\mathrm{x}_{\mathrm{j}}$ are binary since the interest is only to use one time series forecast. The problem is then formulated with objective is to minimize the deviation from an $\mathrm{ME}=0$ on aggregate level, while minimizing the deviation from the lowest SDE for products $i$. Equal weights of goals is used in this example, so the normalization constraint is not strictly necessary:

S.t.

$$
\begin{aligned}
& \quad \text { Minimize: } \quad w_{k} \sum_{j} d_{j^{+}}+w_{k} \sum_{i} \sum_{j} d_{j i^{+}} \\
& \sum_{k} w_{k}=1 \\
& M E_{j} \cdot x_{j}-d_{j^{+}}+d_{j^{-}}=0 \\
& \sum_{j} x_{j}=1 \\
& \left(S D E_{j i}-S D E_{\min , i}\right) \cdot x_{j}-d_{j i^{+}}=0 \\
& x_{j}, d_{j^{+}}, d_{j^{-}}, d_{j i^{+}} \geq 0
\end{aligned}
$$

It can easily be seen that this is a weighted G-MILP problem. Furthermore, $\mathrm{d}_{\mathrm{j}^{+}}, \mathrm{d}_{\mathrm{j}^{-}}$ and $\mathrm{d}_{\mathrm{ji}}+$ are zero for $\mathrm{x}_{\mathrm{j}}=0$, thereby ensuring that only one aggregate forecast is used. Conducting the optimization yields the results as seen in table 2 .

Table 2. Resulting decision and deviation variables values for optimum, with equal weights to $\mathrm{ME}=0$ and sum of SDE.

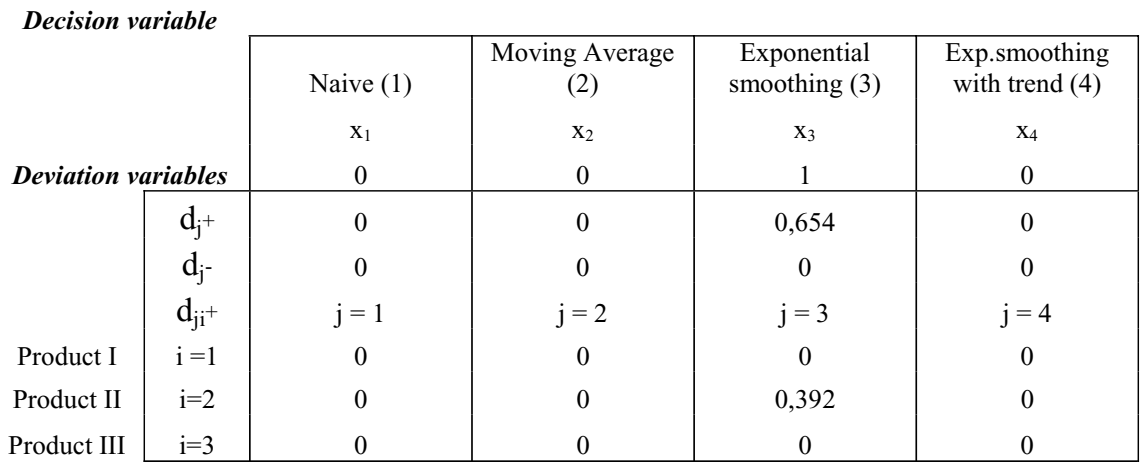


Another approach could be to assign a particular weight to one of the $\mathrm{d}_{\mathrm{ji}}{ }^{+}$ variables. This would be prudent if e.g. one product was significantly more expensive than the two others. The weights will be addressed in the discussion paragraph.

The binary constraint can be relaxed to achieve a linear combination of forecasts, this however requires a dynamic recalculation of forecast error for the linear combination forecasts as well as the introduction of three new deviation variables. The need for a dynamic recalculation of the forecast error SDE makes the problem non-linear. If only $\mathrm{ME}=0$ was used as the goal, the problem would be a simple LP problem, there would however be a risk of multiple solutions with widely diverging $\mathrm{SDE}_{\mathrm{i}}$ values. Moreover, the deviation from $\mathrm{ME}_{\mathrm{ji}}=0$ might be included in a number of situations, see following paragraph.

\section{Discussion}

The way that DP is handled varies greatly depending on the assumed perspective. Otto and Kotzab [12] argue that six perspectives on SCM exist: System Dynamics, Operations Research (OR/IT), Logistics, Marketing, Organization and Strategy. The metrics and consequent criteria for success naturally depend on the assumed perspective. More critically however, is that the criteria for success often seem to conflict - e.g. from the System Dynamics' perspective; capacity utilization and from Logistics perspective; inventory levels. This issue is particular important in connections with HDP.

A number of inferences can be reached from the use of GP to find the optimal forecast on aggregate data, when simple distribution keys are used and the data on the disaggregate level is interdependent. Firstly, the approach is not perfect, but it is simple and cost effective and will fit well into most companies DPC processes. Secondly, it is apparent that the ability to balance these diverging goals is a needed extension of the HDPA. This goes not only for reaching an optimal and feasible plan on more than one planning level, but also for the information to be used to achieve this plan. The simple approach presented here addresses this through balancing the need for a given forecasting performance on aggregate level with the need for a given performance on a disaggregate level. As seen in the GP approach to balance different types of forecast errors in hierarchical forecasting some guidelines for how to weight the error types in a DP hierarchy must be established. As a starting point the critical planning areas must be identified, e.g. to match capacity internally or upstream in the supply chain (focus on $\mathrm{ME}_{\text {aggregate}}$ ) or to find critical products (focus on $\mathrm{ME}_{\text {products }}$ and/or $\mathrm{SDE}_{\text {products }}$ ). $\mathrm{ME}=0+$ (bias to overshoot) would be a problem if capacity is scarce, $\mathrm{ME}=0$ - (bias to undershoot) is a problem when capacity is expensive - since too much capacity would be reserved compared to the average needs. If capacity is not an issue compared to say the price of the products being produced, the SDE of the individual products should be weighted higher. When a balance has been achieved between the relative importance of matching capacity on an aggregate level precisely compared to achieving suitable forecasts for inventory management, the next step is to weight the relative importance of the products within 
a given product family. Two dimensions typically need to be addressed, respectively the relative price and volume of the products [6]. However, dimensions such as customer size and criticality of products/components might be more important in some DPC situations. Assuming price and volume are critical some guidelines for weights are shown below.

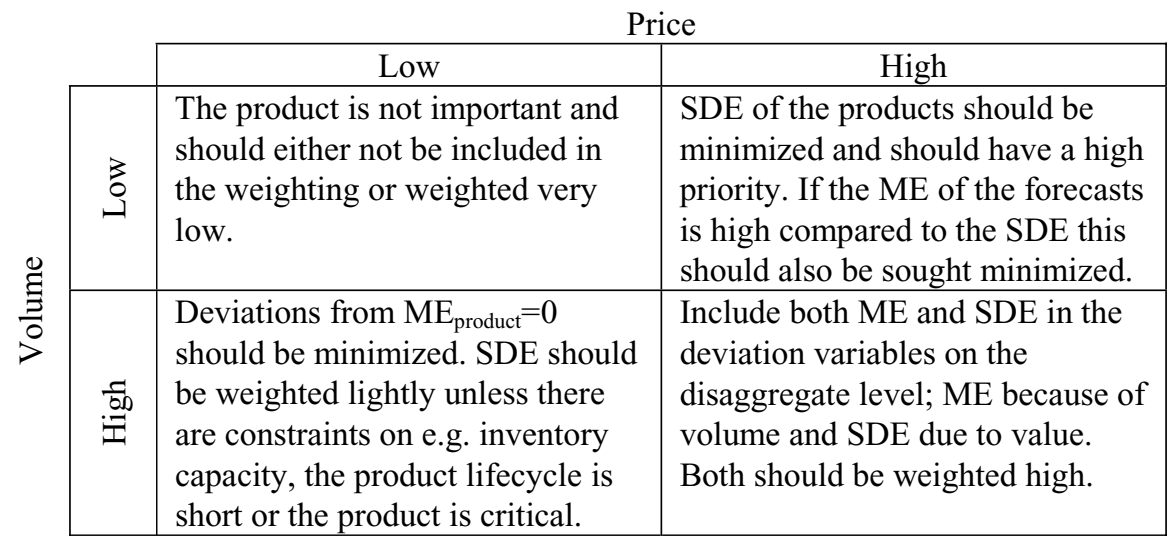

These weights could be quantitatively established using a simple spreadsheet by taking the combined relative volume and price and use these and then normalize these so that the weights between aggregate and disaggregate levels match.

The presented method can with some work be utilized in the hierarchical forecasting procedures treated in e.g. Fliedner [5] so that forecasts on both aggregated and disaggregated data are used to arrive at forecasts for both the aggregate and disaggregate level. The goal would still be to balance the deviation from ME equal to zero and minimize SDE on the disaggregate level. However, solving this problem would entail using non-linear optimization techniques. Furthermore, it is not immediately apparent whether this would be a convex problem.

\section{Conclusion and further research}

Based on the example it is concluded that it is possible to achieve a balance between aggregate and disaggregate forecasts in a hierarchy of forecasts. This is done by using distribution keys on an aggregate forecast and then through GP finding the aggregate forecast that best matches the objective of ME equaling zero on the aggregate level and has a minimum SDE on the disaggregate level. The paper addresses the important issue of being conscious of the limitations in the hierarchical demand planning approach. Moreover, it presents a simple holistic way of dealing with this tradeoff using G-MILP. 
However, further research should take another approach to this issue. Basically the HDPA is flawed in its conception of independence between objects and objectives. Products are typically not independent with regards to sales within a product family, product families are typically not independent of each other and so on. The conclusion is that it is necessary to rethink the methods behind the data used within the HDPA through a new paradigm. The way forward must be to use a data structure and planning methods that include and utilize these interdependencies. One approach that seems to be probable to yield results would be multivariate analysis methods using folded distributions of sales data, which includes the covariance between e.g. product families. If such a method could be developed, then the assumption of independence within the HDPA could be relaxed, yielding a method that more closely mimics the reality of Manufacturing Planning and Control.

\section{References:}

1. Bitran, G. R., E. A. Haas, and A. C. Hax, Hierarchical Production Planning: A Single Stage System, Operations Research, 29, pp. 717-743, 1981.

2. Bitran, G. R., E. A. Haas, and A. C. Hax, Hierarchical Production Planning: A Two Stage Approach, Operations Research, 30, pp. 232-251, 1982.

3. Bitran, G. R. and A. C. Hax, On the design of hierarchical production planning and inventory control systems, Bulletin of the Operations Research Society of America, 23, 1975.

4. Chankong, V., and Y. Y. Haimes, "Multiobjective decision making: theory and methodology", North-holland series in System Science and Engineering; 8, Elsevier Science, 1983.

5. Fliedner, G., Hierarchical forecasting: issues and use guidelines, Industrial Management \& Data Systems, 101, pp. 5-12, 2001.

6. Flores, B. E., and D. C. Whybark, Multiple Criteria ABC Analysis, International Journal of Operations \& Production Management, 6, pp. 38-46, 1986.

7. Hax A. C. and J. J. Golovin, Hierarchical production planning systems, Studies in Operations Management, North-Holland, 1978.

8. Hax, A. C., and H. C. Meal, Hierarchical Integration of Production Planning and Scheduling, in Logistics, edited by M. A. Geisler, vol. 1 of Studies in the Management Sciences, North-Holland/American Elsevier, 1975.

9. Hendry, L. C. and B. G. Kingsman, Production Planning Systems and Their Applicability to Make-to-Order Companies, European Journal of Operational Research, 6, pp. 1-15, 1989.

10. Ignizio, J. P., Goal Programming and Extensions, Lexington Books, 1976. 
11. Lee, S. M., Goal Programming for Decision Analysis, First edition, Auerbach Publishers Inc., 1972.

12. Otto, A., and H. Kotzab, Does supply chain management really pay? Six perspectives to measure the performance of managing a supply chain, European Journal of Operational Research, 144, pp. 306-320, 2003.

13. Silver, E. A., D. F. Pyke, and R. Peterson, Inventory Management and Production Planning and Scheduling, third ed., John Wiley \& Sons, 1998.

14. Theil, H., Applied Economic Forecasting, vol. 4 of Studies in Mathematical and Managerial Economics, North-Holland Publishing Company, 1966.

15. Vollmann, T., W. Berry, and D. Whybark, Manufacturing Planning and Control Systems, fourth ed., Irwin/McGraw-Hill, 1997. 